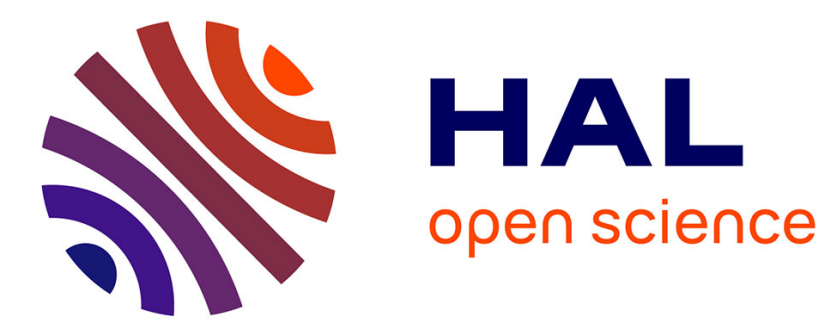

\title{
On the 2-resonance of fullerenes
}

Tomas Kaiser, Matej Stehlík, Riste Škrekovski

\section{To cite this version:}

Tomas Kaiser, Matej Stehlík, Riste Škrekovski. On the 2-resonance of fullerenes. SIAM Journal on Discrete Mathematics, 2011, 25 (4), pp.1737-1745. 10.1137/10078699X . hal-00676969

\section{HAL Id: hal-00676969 https://hal.science/hal-00676969}

Submitted on 6 Mar 2012

HAL is a multi-disciplinary open access archive for the deposit and dissemination of scientific research documents, whether they are published or not. The documents may come from teaching and research institutions in France or abroad, or from public or private research centers.
L'archive ouverte pluridisciplinaire HAL, est destinée au dépôt et à la diffusion de documents scientifiques de niveau recherche, publiés ou non, émanant des établissements d'enseignement et de recherche français ou étrangers, des laboratoires publics ou privés. 


\title{
On the 2-resonance of fullerenes*
}

\author{
Tomáš Kaiser ${ }^{\dagger} \quad$ Matěj Stehlík ${ }^{\ddagger} \quad$ Riste Škrekovski ${ }^{\S}$
}

September 5, 2011

\begin{abstract}
We show that every pair of hexagons in a fullerene graph satisfying the isolated pentagon rule (IPR) forms a resonant pattern. This solves a problem raised by Ye et al. [SIAM J. Discrete Math. 23(2):2009, p. 1023-1044].
\end{abstract}

\section{Introduction}

A fullerene graph is a 3-connected cubic plane graph with all faces of size 5 and 6 . Fullerene graphs satisfying the isolated pentagon rule-those with no adjacent pentagonal faces - are known as IPR fullerene graphs.

Fullerene graphs serve as models for fullerene molecules, discovered in 1985 by Kroto et al. [8]. IPR fullerenes are of particular interest, since they appear to be the most stable. Perfect matchings in fullerene graphs correspond to Kekulé structures in fullerenes, and as such have received much attention. For more information on fullerenes, we refer the reader to the monograph of Fowler and Manolopoulos [3].

Let $M$ be a perfect matching in a fullerene graph $G$. An even cycle of $G$ is $M$-alternating if exactly half its edges are in $M$. A set of disjoint $M$ alternating hexagons of $G$ is called a resonant pattern. A fullerene graph is $k$-resonant if any $i$ disjoint hexagons form a resonant pattern, for $0 \leq i \leq k$.

*Research supported by the bilateral Czech-Slovenian project MEB090805.

${ }^{\dagger}$ Institute for Theoretical Computer Science (ITI) and Department of Mathematics, University of West Bohemia, Univerzitní 8, 30614 Plzeň, Czech Republic (kaisert@kma.zcu.cz). Supported by project 1M0545 of the Czech Ministry of Education and project GAČR 201/09/0197 of the Czech Science Foundation.

$\ddagger$ UJF-Grenoble 1 / CNRS / Grenoble-INP, G-SCOP UMR5272, Grenoble, F-38031, France (matej.stehlik@g-scop.inpg.fr). The author gratefully acknowledges the support of National Sun Yat-sen University, Taiwan, while writing this paper.

$\S$ Department of Mathematics, University of Ljubljana, Jadranska 19, 1111 Ljubljana, Slovenia. 
Ye et al. [15] proved that every fullerene graph is 1-resonant, and that every leapfrog fullerene graph is 2-resonant (see [15] for the definition). The class of leapfrog fullerene graphs satisfies the IPR; this led Ye et al. [15, Open Problem 3.5] to ask whether every IPR fullerene graph is 2-resonant. Here we answer this question in the affirmative by proving the following result.

Theorem 1. Every IPR fullerene graph is 2-resonant.

The theorem is best possible in the sense that no fullerene graph on more than sixty vertices is 3-resonant, as pointed out by Ye et al. [15].

\section{Notation and preliminary results}

Given a graph $G$, we let $V(G)$ and $E(G)$ be its vertex set and edge set, respectively. For $H \subseteq V(G) \cup E(G)$, we let $G-H$ be the graph obtained from $G$ by removing the elements in $H$. Let $X, X^{\prime} \subset V(G)$. We define $G[X]$ to be the subgraph of $G$ induced by the vertices in $X$. We let $\partial(X)$ be the set of edges of $G$ with exactly one end-vertex in $X$; if $F=G[X]$ we may also write $\partial(F)$ for $\partial(X)$. We denote by $E(X, Y)$ the set of edges with one endvertex in $X$ and the other endvertex in $Y$; if $X=Y$ we simply write $E(X)$. We denote $|E(X, Y)|$ and $|E(X)|$ by $e(X, Y)$ and $e(X)$, respectively.

We say that two faces of a plane embedding of $G$ are adjacent if they share an edge. Vertices and edges contained in the boundary of a face $F$ are said to belong to $F$ or to be on $F$. Pentagonal and hexagonal faces are referred to simply as pentagons and hexagons.

A graph $G$ is factor-critical if $G-v$ has a perfect matching, for every vertex $v \in V(G)$. It is easy to see that every factor-critical graph is connected, has an odd number of vertices, and has minimum degree two unless it consists of a single vertex. Lovász [10] characterised factor-critical graphs as those having an 'odd ear decomposition' (see also [11, 12]). The definition of factor-criticality implies that a factor-critical graph is 2-edge-connected. Using the fact that fullerenes are cubic graphs, we obtain the following slightly stronger conclusion:

Observation 2. Every non-trivial factor-critical subgraph of a fullerene graph is 2-connected.

The following theorem may be viewed as a strengthening of Tutte's 1factor theorem [14]. It is a simple corollary of the Gallai-Edmonds decomposition theorem [2, 4, 5] (see also [11, 12]).

Theorem 3. A graph $G$ has no perfect matching if and only if there exists a subset $A \subseteq V(G)$ such that: 

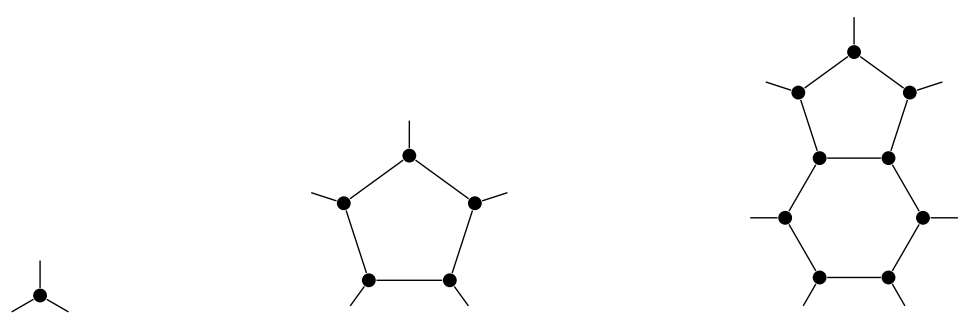

Figure 1: Cases (i)-(iii) in Lemma 4.

(i) every component of $G-A$ has a perfect matching or is factor-critical, and

(ii) at least $|A|+1$ components of $G-A$ are factor-critical.

A $k$-edge-cut of $G$ is a set $Y$ of $k$ edges of $G$ for which there exists a set $X \subset V(G)$ such that $Y=\partial(X)$. A $k$-edge-cut $Y$ is cyclic if at least two components of $G-Y$ contain a cycle. The graph $G$ is cyclically $k$-edgeconnected if $|E(G)|>k$, and it contains no cyclic edge-cuts consisting of fewer than $k$ edges.

Došlić [1] proved that fullerene graphs are cyclically 5-edge connected. Kardoš and Škrekovski [7] characterised the cyclic 5- and 6-edge-cuts in fullerene graphs; the cyclic 5-edge-cuts were characterised independently by Kutnar and Marušič [9]. Furthermore, Kardoš et al. [6] characterised the cyclic 7-edge-cuts in fullerene graphs. The characterisations together imply the following three lemmas (see Figures 13 for illustrations). The proofs are omitted, as they amount to verifying which configurations satisfy the IPR and are factor-critical, resp. have a perfect matching. For a subgraph $F$ of a graph $G$, the symbol $F^{c}$ stands for $G-V(F)$.

Lemma 4. Let $F$ be a factor-critical subgraph of an IPR fullerene graph. Then one of the following assertions holds.

(i) $|\partial(F)|=3$ and $F$ or $F^{c}$ consists of a single vertex;

(ii) $|\partial(F)|=5$ and $F$ or $F^{c}$ is a pentagon;

(iii) $|\partial(F)|=7$ and $F$ or $F^{c}$ consists of a pentagon and an adjacent hexagon;

(iv) $|\partial(F)| \geq 9$. 

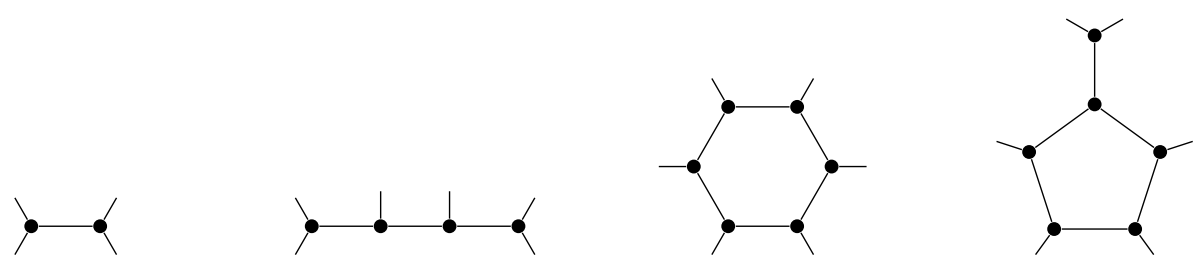

Figure 2: Case (i) and the three subcases of case (ii) in Lemma 5.
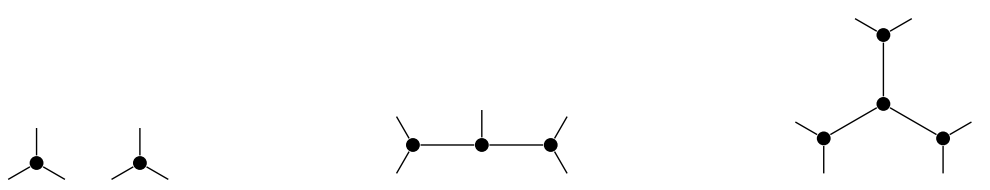

Figure 3: The three new cases in Lemma 6. The remaining possibilities are the first two graphs in Figure 1 and the graphs in Figure 2.

Lemma 5. Let $F$ be a subgraph with a perfect matching of an IPR fullerene graph. Then one of the following assertions holds.

(i) $|\partial(F)|=4$ and $F$ or $F^{c}$ is isomorphic to $K_{2}$;

(ii) $|\partial(F)|=6$ and $F$ or $F^{c}$ is a path of length 3 , a hexagon, or a pentagon with a pendant edge;

(iii) $|\partial(F)| \geq 8$.

We will also need to extend the characterisations of Lemmas 4 and 5 to subgraphs $F$ with $|\partial(F)| \leq 6$ that are not necessarily factor-critical and may have no perfect matching. There are only three additional cases (cf. Figure 3):

Lemma 6. Let $F$ be a subgraph of an IPR fullerene with $|\partial(F)| \leq 6$. Then $F$ or $F^{c}$ is either a pair of non-adjacent vertices, a path of length at most 3, a $K_{1,3}$, a cycle of length 5 or 6 , or a pentagon with a pendant edge.

\section{The proof of Theorem 1}

Before embarking on the proof of Theorem 1, let us give a brief outline of the main argument, which is a substantial refinement of those in [13, 15]. 


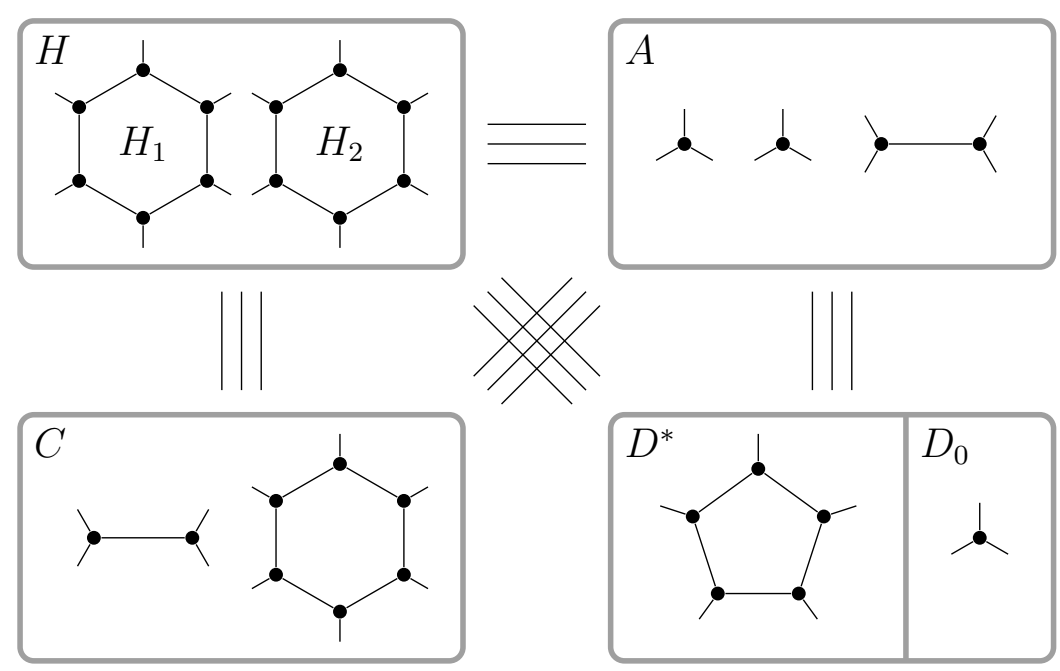

Figure 4: The partition of $V(G)$ into $H, A, C, D^{*}$ and $D_{0}$.

Using Theorem 3, we show that deleting any pair of disjoint hexagons from a fullerene graph results in a graph which is 'almost bipartite', in the sense that deleting a prescribed set of vertices and edges gives a bipartite graph. Then, using Lemmas 4 and 5 we show that, apart from one special case, there is always at least one pentagon which does not intersect this prescribed set; a contradiction. The remainder of the proof is then dedicated to ruling out the special case.

Proof of Theorem 1. Let $G$ be an IPR fullerene graph, let $H_{1}$ and $H_{2}$ be two disjoint hexagons of $G$, and set $H:=V\left(H_{1} \cup H_{2}\right)$. Suppose $G-H$ does not have a perfect matching. Then Theorem 3 guarantees the existence of a set $A$ of vertices of $G-H$ such that $G-(H \cup A)$ contains more than $|A|$ factor-critical components, and all other components of $G-(H \cup A)$ have a perfect matching. We denote the factor-critical components of $G-(H \cup A)$ by $\mathcal{D}$, and the components of $G-(H \cup A)$ with a perfect matching by $\mathcal{C}$. The subset of non-trivial factor-critical components of $G-(H \cup A)$ is denoted by $\mathcal{D}^{*}$. The union of the vertex sets of the components of $\mathcal{C}, \mathcal{D}$ and $\mathcal{D}^{*}$ is denoted by $C, D$ and $D^{*}$, respectively, and we set $D_{0}:=D-D^{*}$ (see Figure 4).

The number of vertices of $G$ being even, $|\mathcal{D}|$ and $|A|$ have the same parity. Since $|\mathcal{D}|>|A|$, we deduce that

$$
|\mathcal{D}| \geq|A|+2
$$

The number of edges leaving $A \cup H$ may be expressed as

$$
|\partial(A \cup H)|=|\partial(H)|+3|A|-2 e(A, H)-2 e(A) .
$$


Now set

$$
s(\mathcal{D}):=\sum_{F \in \mathcal{D}}(|\partial(F)|-3) / 2
$$

and

$$
s(\mathcal{C}):=\sum_{F \in \mathcal{C}}(|\partial(F)|-4) / 2 .
$$

By Lemmas 4 and 5 , the number of edges leaving $C \cup D$ may be expressed as

$$
|\partial(C \cup D)|=3|\mathcal{D}|+4|\mathcal{C}|+2(s(\mathcal{C})+s(\mathcal{D})) .
$$

Since $\partial(A \cup H)=\partial(C \cup D),(1),(2)$ and (3) imply that

$$
2|\mathcal{C}|+e(A, H)+e(A)+s(\mathcal{C})+s(\mathcal{D}) \leq \frac{1}{2}|\partial(H)|-3 \leq 3 .
$$

Let $\mathcal{P}$ be the set of pentagons of $G$; it follows from Euler's formula that $|\mathcal{P}|=12$. If $X \subseteq V(G)$ and $Y \subseteq E(G)$, let $p(X):=\mid\{P \in \mathcal{P} \mid V(P) \cap X \neq$ $\emptyset\} \mid$ and $p(Y):=|\{P \in \mathcal{P} \mid E(P) \cap Y \neq \emptyset\}|$.

Observe that every edge of the graph $G-\left(H \cup C \cup D^{*} \cup E(A)\right)$ has one endvertex in $A$ and the other in $D_{0}$; in particular, $G-\left(H \cup C \cup D^{*} \cup E(A)\right)$ is bipartite. This means that all twelve pentagons of $G$ must contain a vertex in $H \cup C \cup D^{*}$ or an edge in $E(A)$. By the IPR, $p(H) \leq 6$, so at least six pentagons disjoint from $H$ must have a vertex in $C \cup D^{*}$ or an edge in $E(A)$. In particular,

$$
p(E(A))+p(C)+p\left(D^{*}\right) \geq 6
$$

Claim 1. There exists a component $F^{*} \in \mathcal{D}^{*}$ such that $\left|\partial\left(F^{*}\right)\right|=9$.

By (4), $|\mathcal{C}| \leq 1$. If $|\mathcal{C}|=1$, then $e(A)+s(\mathcal{D})+s(\mathcal{C}) \leq 1$ by (4). By the IPR, $p(E(A)) \leq e(A) \leq 1$. As $s(\mathcal{D}) \leq 1$, Lemma 4 and the IPR imply that $p\left(D^{*}\right) \leq 1$. Finally, by Lemma 5 and the IPR, $p(C) \leq s(\mathcal{C})+2 \leq 3$. Hence $p(E(A))+p(C)+p\left(D^{*}\right) \leq e(A)+s(\mathcal{D})+s(\mathcal{C})+2<6$, contradicting (5).

Hence we may assume that $\mathcal{C}=\emptyset$. In particular, $p(C)=s(\mathcal{C})=0$, and by (4) $e(A)+s(\mathcal{D}) \leq 3$. This implies that $|\partial(F)| \leq 9$ for every $F \in \mathcal{D}^{*}$. If $|\partial(F)|<9$ for every $F \in \mathcal{D}^{*}$, then by Lemma 4 and the IPR, $p\left(D^{*}\right) \leq s(\mathcal{D})+$ 1. Hence $p(E(A))+p(C)+p\left(D^{*}\right) \leq e(A)+s(\mathcal{D})+1<6$, contradicting (5). Therefore $\left|\partial\left(F^{*}\right)\right|=9$ for some component $F^{*} \in \mathcal{D}^{*}$, as claimed.

By (4), $F^{*}$ is the unique non-trivial component of $\mathcal{D}, \mathcal{C}=\emptyset, e(A)=0$ (so $A$ is an independent set), $e(A, H)=0$ and $|\partial(H)|=12$ (in particular, there are no edges between the hexagons $H_{1}$ and $H_{2}$ ). As $F^{*}$ is non-trivial 


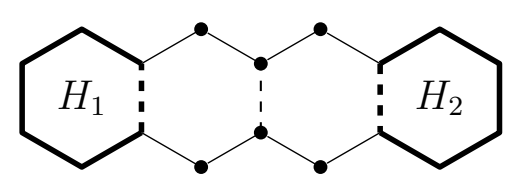

Figure 5: A link of size 3. The hexagons $H_{1}$ and $H_{2}$ are drawn bold, the edges of the link are dashed.

and factor-critical, it is 2-connected by Observation 2. In particular, it has minimum degree 2 .

Let us now fix a planar embedding of $G$. If $e, f$ are edges of $G$, we will say that $e$ is opposite $f$ if they both belong to the boundary of some hexagonal face and no edge of this boundary is incident with both $e$ and $f$. A link is a sequence $L=\left(e_{1}, \ldots, e_{k}\right)$ of edges, where

(1) $e_{i+1}$ is opposite $e_{i}$ for $i=1, \ldots, k-1$,

(2) $e_{1}$ belongs to $H_{1}$ and $e_{k}$ belongs to $H_{2}$.

(See Figure 5 for an illustration.) The size $|L|$ of such a link is defined as $k$. The following observation is immediate:

Observation 7. Any two links in $G$ are disjoint.

We continue with a simple structural lemma.

Lemma 8. $G$ contains no two links $L_{1}, L_{2}$ with $\left|L_{1}\right|+\left|L_{2}\right| \leq 6$.

Proof. Suppose such $L_{1}$ and $L_{2}$ exist. We claim that their union (as sets) forms an edge-cut of $G$. This is easy to see from the duality between edgecuts in $G$ and cycles in the dual graph. For a direct argument, note that one can draw a closed curve $C$ in the plane which only intersects the edges in $L_{1} \cup L_{2}$ (connecting each pair of opposite edges by a line segment through the face containing them, and proceeding similarly on $H_{1}$ and $H_{2}$ ). In addition, the subgraphs $G_{1}$ and $G_{2}$ into which $G$ is divided by $C$ can easily be seen to be connected, which shows that $L_{1} \cup L_{2}$ is an edge-cut.

Lemma 6 gives us the possibilities for $G_{1}$ or $G_{2}$. Let us assume that $G_{1}$ is one of the subgraphs listed in the lemma. Let $N$ be the set of faces of $G$ which intersect both $G_{1}$ and $G_{2}$. For each face $F$ in $N$, let $\ell(F)$ be the number of edges of $G_{1}$ that belong to $F$. By the definition of a link, we have $\ell(F)=2$ for each $F \in N \backslash\left(H_{1} \cup H_{2}\right)$. However, considering the possibilities for $G_{1}$ of Lemma 6 (with two different embeddings in the case of the path of length 3) we find that in each case, at least three values of $\ell(F)$ are different from 2. See Figure 6 for an illustration. This contradiction concludes the proof. 

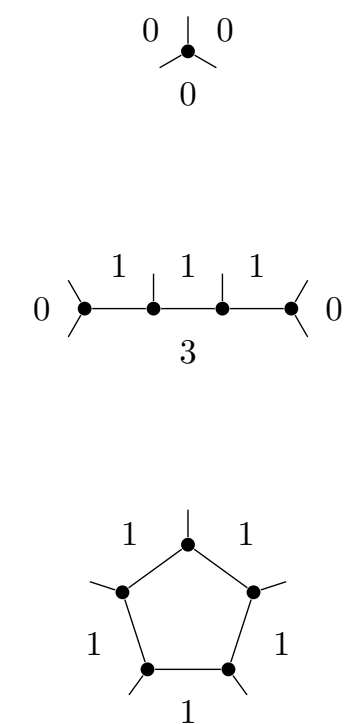
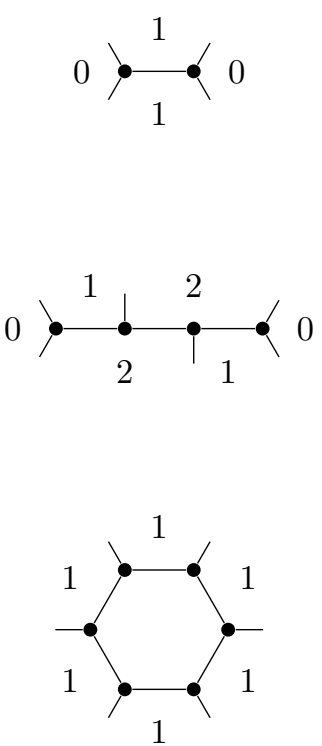
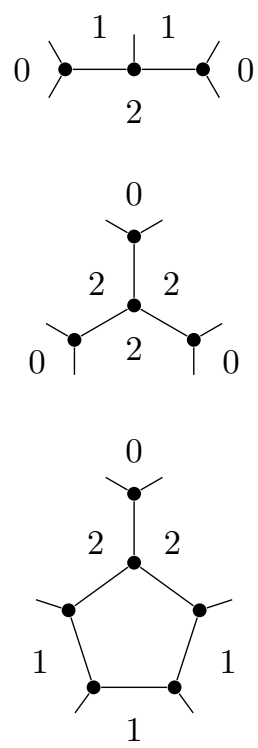

Figure 6: The values of $\ell(F)$ in the proof of Lemma 8 (shown as the numbers inside the faces adjacent to $\left.G_{1}\right)$.

The following lemma is the core of our argument.

Lemma 9. Let $j \in\{1,2\}$. Assume that $a, b$ are adjacent vertices of $H_{j}$ and $M$ is the face of $G$ whose boundary includes the edge $a b$ and which is different from $H_{j}$. Let $a^{\prime}, b^{\prime}$ be the neighbours of $a$ and $b$ (respectively) not in $H_{j}$. If neither $a a^{\prime}$ nor $b b^{\prime}$ is an edge of $\partial\left(F^{*}\right)$, then $M$ is either a pentagonal face, or a hexagonal face intersecting both $H_{1}$ and $H_{2}$.

Proof. Suppose that $M$ has length 6, so its boundary is $a^{\prime} a b b^{\prime} x y$, where $x, y \in V(G)$. Since $a^{\prime}$ and $b^{\prime}$ belong neither to $A \cup V\left(H_{1} \cup H_{2}\right)$ nor to $F^{*}$ (by assumption), we have $a^{\prime}, b^{\prime} \in D_{0}$. Thus, $x$ and $y$ are contained in $A \cup V\left(H_{1} \cup H_{2}\right)$. Since $E\left(A \cup V\left(H_{1}\right) \cup V\left(H_{2}\right)\right)=E\left(V\left(H_{1}\right)\right) \cup E\left(V\left(H_{2}\right)\right.$, both $x$ and $y$ must be contained in the same hexagon $H_{k}$ (for some $k=1,2$ ). However, if $x, y \in V\left(H_{j}\right)$, then it is easy to find a cycle of length at most 4 in $G$, contradicting the fact that $G$ is cyclically 5 -edge-connected. Thus, $M$ intersects both $H_{1}$ and $H_{2}$ which proves the claim.

We want to be able to bound the number of edges of $\partial\left(F^{*}\right)$ 'near' $H_{1}$ and $H_{2}$. We will make use of the following lemma.

Lemma 10. Let $F$ be a face of $G$ incident with $H_{j}(j=1,2)$. Then the intersection of $\partial\left(F^{*}\right)$ with the boundary of $F$ is either empty, or it is a matching 
of size 2 which does not form a pair of opposite edges. If, in addition, $F$ is a pentagon, then both edges of the matching have one endvertex on $H_{j}$.

Proof. If the intersection of $\partial\left(F^{*}\right)$ with the boundary of $F$ is non-empty, then it has to be a matching $\{v w, x y\}$, by Observation 2 and the fact that the intersection of a cycle and an edge cut is even. If both $v w$ and $x y$ have a vertex in $V\left(H_{j}\right)$, then $u v, x y$ are not opposite, as required.

So suppose now that one of the edges $v w, x y$ has no vertex in $V\left(H_{j}\right)$; without loss of generality say it is $v w$. If $v$ is adjacent to a vertex of $H_{j}$, then $v \in D_{0}$ and $w \in D^{*}$, contradicting the fact that there are no edges between $D_{0}$ and $D^{*}$. Similarly for $w$. Therefore $v, w$ are not adjacent to any vertex of $V\left(H_{j}\right)$, so $M$ is a hexagon and $v w, x y$ are not opposite.

A cluster at $H_{j}(j=1,2)$ is a sequence $Q=\left(e_{1}, \ldots, e_{k}\right)$ such that:

- each $e_{i}$ is in $\partial\left(F^{*}\right)$,

- $e_{i}$ and $e_{i+1}$ are on the same face $(1 \leq i \leq k-1)$,

- for $2 \leq i \leq k-1$, each $e_{i}$ has exactly one endvertex on $H_{j}$, while $e_{1}$ and $e_{k}$ are not incident with $H_{j}$.

Examples of clusters are shown in Figure 7. The size $|Q|$ of $Q$ is the number of its edges. Observe that $|Q| \geq 3$.

\section{Lemma 11.}

(i) Clusters at $H_{j}$ are pairwise disjoint (for $j=1,2$ ).

(ii) $G$ contains at most one edge $e$ that is contained in a cluster at $H_{1}$ and a cluster at $\mathrm{H}_{2}$. If e exists, then it is contained in a link of size 3 .

Proof. (i) If an edge $e$ belongs to two distinct clusters $C, C^{\prime}$ at $H_{j}$, then it is clearly not incident with $H_{j}$. We may thus assume that $C$ and $C^{\prime}$ are of the form $C=\left(e, e_{2}, \ldots, e_{k}\right)$ and $C^{\prime}=\left(e, e_{2}^{\prime}, \ldots, e_{\ell}^{\prime}\right)$. By the definition of a cluster and Lemma 10, $e$ belongs to hexagonal faces $M$ and $M^{\prime}$ whose boundaries contain $e_{2}$ and $e_{2}^{\prime}$, respectively. However, $e$ belongs to the boundary of only one face adjacent to $H_{j}$, so $M=M^{\prime}$ and this face boundary contains $e, e_{2}$ and $e_{2}^{\prime}$, a contradiction to Lemma 10 .

(ii) Assume an edge $e$ is contained in a cluster $C_{1}$ at $H_{1}$ and $C_{2}$ at $H_{2}$. By Lemma 10 and the fact that there are no edges between $H_{1}$ and $H_{2}$, it is easy to check that $e$ is incident with neither $H_{1}$ nor $H_{2}$. By Lemma $10 e$ is opposite an edge of $H_{1}$ and opposite an edge of $H_{2}$, so it is contained in a link of size 3. The uniqueness of $e$ follows by Lemma 8 . 

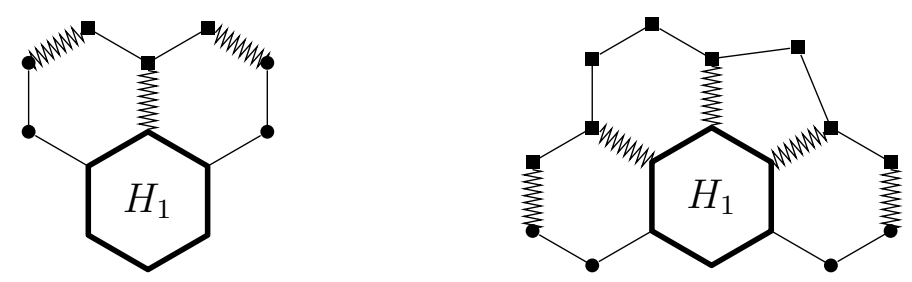

Figure 7: Examples of clusters at $H_{1}$. Left: a cluster of size 3. Right: a cluster of size 5 . The edges of the clusters are shown as zigzag lines, those of $H_{1}$ are drawn bold. Squares represent the vertices of $F^{*}$.

We shall now use Lemma 9 to lower-bound the number of edges of $\partial\left(F^{*}\right)$ incident with each $H_{j}$. Let us call an edge $e$ of $G$ free if $e \notin \partial\left(F^{*}\right)$.

Lemma 12. Let $j \in\{1,2\}$.

(i) $\partial\left(H_{j}\right)$ does not contain five free edges.

(ii) If $\partial\left(H_{j}\right)$ contains three consecutiv@ free edges, then there is a link of size 2 in $G$.

(iii) There is exactly one cluster at $H_{j}$, and its size is 4 or 5 .

Proof. (i) Any set of five free edges in $\partial\left(H_{j}\right)$ contains four consecutive pairs of free edges. By Lemma 9, each of these pairs belongs to the boundary of either a pentagonal face, or a hexagonal face incident with $H_{1}$ and $H_{2}$. Lemma 8 implies that only one of the four pairs is of the latter type. It follows that $\partial\left(H_{j}\right)$ contains three edges $e, e^{\prime}, e^{\prime \prime}$ such that each of the pairs $e, e^{\prime}$ and $e^{\prime}, e^{\prime \prime}$ belongs to a pentagon. Since this would violate the IPR, $\partial\left(H_{j}\right)$ does not contain five free edges.

(ii) This is a direct consequence of Lemma 9 and the IPR.

(iii) Suppose that $\partial\left(H_{j}\right) \subseteq \partial\left(F^{*}\right)$, or that $H_{j}$ is incident with two or more clusters. In both cases there are at most three edges of $\partial\left(F^{*}\right)$ left for the clusters at $H_{3-j}$. Hence there is only one cluster (of size 3 ) at $H_{3-j}$ and five edges of $\partial\left(H_{3-j}\right)$ are free, contradicting (i). The claim follows.

We now use Lemma 12(iii) to conclude the analysis. Assume first that there is a cluster of size 5 at both $H_{1}$ and $H_{2}$. These clusters have nonempty intersection as $\left|\partial\left(F^{*}\right)\right|$ is only 9. By Lemma 11(ii), $G$ contains a link of size 3. On the other hand, observe that the set $\partial\left(H_{1}\right)$ contains three consecutive free edges, which by Lemma 12 (ii) is only possible if there is a link of size 2 in $G$. The existence of these two links contradicts Lemma 8 ,

\footnotetext{
${ }^{1}$ 'Consecutive' refers to the natural clockwise ordering of $\partial\left(H_{j}\right)$.
} 


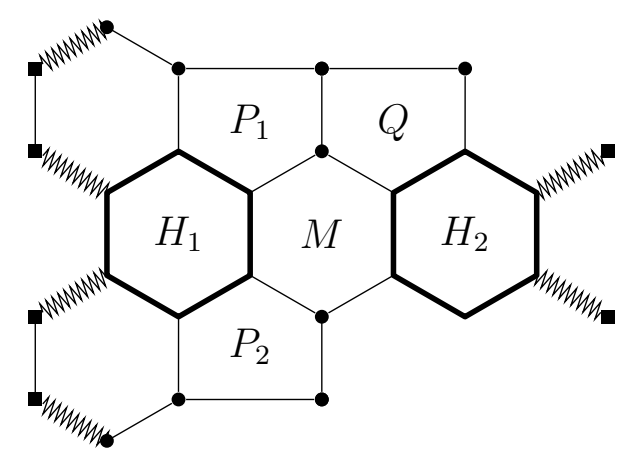

Figure 8: An illustration to the final argument in the proof of Theorem 1 (the case when $Q$ is adjacent to $P_{1}$ ). The conventions for the vertices and edges are the same as in Figure 7 .

We may thus assume that the cluster at $H_{1}$ has size 4 . Since there are four consecutive free edges $e_{1}, \ldots, e_{4} \in \partial\left(H_{1}\right)$, Lemma 12 (ii) implies the existence of a link $L$ of size 2 in $G$. Figure 8 depicts the situation. Let $M$ be the (hexagonal) face whose boundary contains both edges of $L$. Lemma 8 and the fact that there are no edges between the hexagons $H_{1}$ and $H_{2}$ implies that $M$ is the unique face intersecting both $H_{1}$ and $H_{2}$. Therefore, by Lemma 9 and the IPR, $e_{2}$ and $e_{3}$ belong to $M$, while the pairs $e_{1}, e_{2}$ and $e_{3}, e_{4}$ belong to pentagonal faces $\left(P_{1}\right.$ and $P_{2}$, respectively) adjacent to $H_{1}$ and $M$.

The cluster at $H_{2}$ has size 4 or 5 , which means that $\partial\left(H_{2}\right)$ contains at least three consecutive free edges. By another application of Lemma 9 and the IPR, two of them must belong to a pentagonal face $Q$ adjacent to $M$ and $H_{2}$. However, it is easy to see that $Q$ is adjacent to either $P_{1}$ or $P_{2}$. This contradiction to the IPR completes the proof of Theorem 1 .

\section{References}

[1] T. Došlić. Cyclical edge-connectivity of fullerene graphs and $(k, 6)$-cages. J. Math. Chem., 33(2):103-112, 2003.

[2] J. Edmonds. Paths, trees, and flowers. Canad. J. Math., 17:449-467, 1965.

[3] P. W. Fowler and D. E. Manolopoulos. An Atlas of Fullerenes. Oxford University Press, Oxford, 1995.

[4] T. Gallai. Kritische Graphen. II. Magyar Tud. Akad. Mat. Kutató Int. Közl., 8:373-395, 1963. 
[5] T. Gallai. Maximale Systeme unabhängiger Kanten. Magyar Tud. Akad. Mat. Kutató Int. Közl., 9:401-413, 1964.

[6] F. Kardoš, M. Krnc, B. Lužar, and R. Škrekovski. Cyclic 7-edge-cuts in fullerene graphs. J. Math. Chem., 47(2):771-789, 2010.

[7] F. Kardoš and R. Škrekovski. Cyclic edge-cuts in fullerene graphs. J. Math. Chem., 44(1):121-132, 2008.

[8] H. W. Kroto, J. R. Heath, S. C. O'Brien, R. F. Curl, and R. E. Smalley. $\mathrm{C}_{60}$ : Buckminsterfullerene. Nature, 318:162-163, 1985.

[9] K. Kutnar and D. Marušič. On cyclic edge-connectivity of fullerenes. Discrete Applied Math., 156(10):1661-1669, 2008.

[10] L. Lovász. A note on factor-critical graphs. Studia Sci. Math. Hungar., $7: 279-280,1972$.

[11] L. Lovász and M. D. Plummer. Matching theory, volume 121 of NorthHolland Mathematics Studies. North-Holland Publishing Co., Amsterdam, 1986. Annals of Discrete Mathematics, 29.

[12] A. Schrijver. Combinatorial optimization: Polyhedra and efficiency, volume 24 of Algorithms and Combinatorics. Springer-Verlag, Berlin, 2003.

[13] J.-S. Sereni and M. Stehlík. On the sextet polynomial of fullerenes. J. Math. Chem., 47(3):1121-1128, 2010.

[14] W. T. Tutte. The factorization of linear graphs. J. London Math. Soc., 22:107-111, 1947.

[15] D. Ye, Z. Qi, and H. Zhang. On k-resonant fullerene graphs. SIAM J. Discrete Math., 23(2):1023-1044, 2009. 